\title{
Synthesis and Characterization of Iron Oxide Derivatized Mutant Cowpea Mosaic Virus Hybrid Nanoparticles**
}

\author{
By Alfredo A. Martinez-Morales, Nathaniel G. Portney, Yu Zhang, \\ Giuseppe Destito, Gurer Budak, Ekmel Ozbay, Marianne Manchester, \\ Cengiz S. Ozkan, * and Mihrimah Ozkan*
}

Extensively investigated and mutagenized Cow Pea Mosaic Virus (CPMV) has been demonstrated in a variety of nanoassemblies. ${ }^{[1-3]}$ Iron Oxide (IO) has the potential to surpass limits of detection in bioimaging applications. Particularly $\gamma-\mathrm{Fe}_{2} \mathrm{O}_{3}$ (maghemite) is considered as one of the most desirable materials for technological and biomedical applications due to its inherent biocompatible nature. ${ }^{[4,5]}$ Additionally, maghemite nanoparticles could be directed to an organ, tissue, or tumor using an external magnetic field or heated under an alternating magnetic field. ${ }^{[6,7]}$ Based on the unique magnetic properties of IO nanoparticles they have been extensively used in biomedical applications, such as magnetic resonance imaging, targeting drug delivery and hyperthermia therapy detoxification and cell separation. ${ }^{[8-12]}$

[*] Prof. C. S. Ozkan, Y. Zhang

Department of Mechanical Engineering,

University of California Riverside

92521 Riverside, CA (USA)

E-mail: cozkan@engr.ucr.edu

Prof. M. Ozkan, A. A. Martinez-Morales

Department of Electrical Engineering,

University of California Riverside

92521 Riverside, CA (USA)

E-mail: mihri@ee.ucr.edu

Dr. N. G. Portney

Department of Bioengineering, University of California Riverside 92521 Riverside, CA (USA)

Dr. G. Destito, Prof. M. Manchester

Department of Cell Biology, The Scripps Research Institute

92037 La Jolla, CA (USA)

Prof. G. Budak

Faculty of Medicine, Nanomedicine Research Laboratory,

Gazi University

06510 Ankara (Turkey)

Prof. E. Ozbay

Nanotechnology Research Center

Department of Electrical and Electronics Engineering

and Department of Physics

Bilkent University

06800 Ankara (Turkey)

[**] This research was supported by the Center for Nanotechnology for the Treatment, Understanding and Monitoring of Cancer (NANOTUMOR) funded by the National Cancer Institute $(\mathrm{NCl})$ and by the FCRP Center on Functional Engineered Nano Architectonics (FENA) funded by the Defense Advanced Research Projects Agency (DARPA) and the Semiconductor Research Corporation (SRC). The authors gratefully acknowledge Freida Dallal for technical assistance during FTIR studies and Nissim Amos for fruitful discussions on MFM imaging.
Combining the two systems can be devised to enhance the local magnetic field strength, by organizing monodisperse IO clusters on a CPMV-T184C mutant viral template. It is known that contrast enhancement is observed by use of superparamagnetic iron oxide nanoparticles (SPIONs) based MRI, by creating large dipolar magnetic field gradients due to their local field inhomogeneity. However, clustering a greater number of IO nanoparticles can further improve contrast beyond free particle SPIONs enhanced MRI, by creating a cumulative dipole effect. ${ }^{[13]}$

CPMV-T184C is a useful model that has a well characterized structure amenable to surface functionalization. ${ }^{[14]}$ The smallest repeating structure (asymmetric unit, composed of a "small" (24kD) and "large" (42kD) subunit) displays 5 solvent exposed lysines used for IO linkage. ${ }^{[15]}$ By insertion of a cysteine, at residue 184 of the small subunit, anchorage of CPMV to a self assembled monolayer (SAM) on gold substrate pathway can be employed. A previously reported SAM on Au stepwise assembly was used to integrate monodisperse CPMV-IO hybrids for characterization. ${ }^{[16]}$

It is also been shown that aggregation of Iron oxide particles can exhibit a greater magnetic dipole, and can be suited for in bio-imaging, provided certain properties are met. Harris et al., ${ }^{[13]}$ demonstrated protease activated aggregation of pegylated iron oxide nanoparticles with enhanced MRI contrast to be most beneficial in improving detection limits of small tumors. Pegylation of CPMV was previously demonstrated to improve circulation times and reduce immunogenicity. ${ }^{[2]}$ Also, based on enhanced permeability and retention effects (EPR), ${ }^{[17]}$ the longest retention times at tumor sites for nanoparticles occurred for $60-400 \mathrm{~nm} \cdot{ }^{[18]}$ Above $300 \mathrm{~nm}$, there is vulnerability to macrophage phagocytosis, ${ }^{[19]}$ and below $10 \mathrm{~nm}$, nanoparticles can leave the systemic circulation via the lymph nodes. ${ }^{[20]}$ Therefore, the IONs-CPMV nanoparticle hybrid system synthesized and MFM characterized in this report could be used for contrast enhanced MRI applications.

In this work the local enhancement of field strength is studied and demonstrated by magnetic force microscopy (MFM) characterization of CPMV-IO hybrids bound to a substrate by a stepwise assembly process (Fig. 1).

AFM was used to characterize structurally the as-synthesized IO nanoparticles on a silicon substrate (Fig. 2A). In addition, a histogram of the size distribution of the IO nanoparticles (Inset Fig. 2A) determined from 68 individual measurements on single IO nanoparticles exhibited a mean size of $\sim 11 \mathrm{~nm}$. 


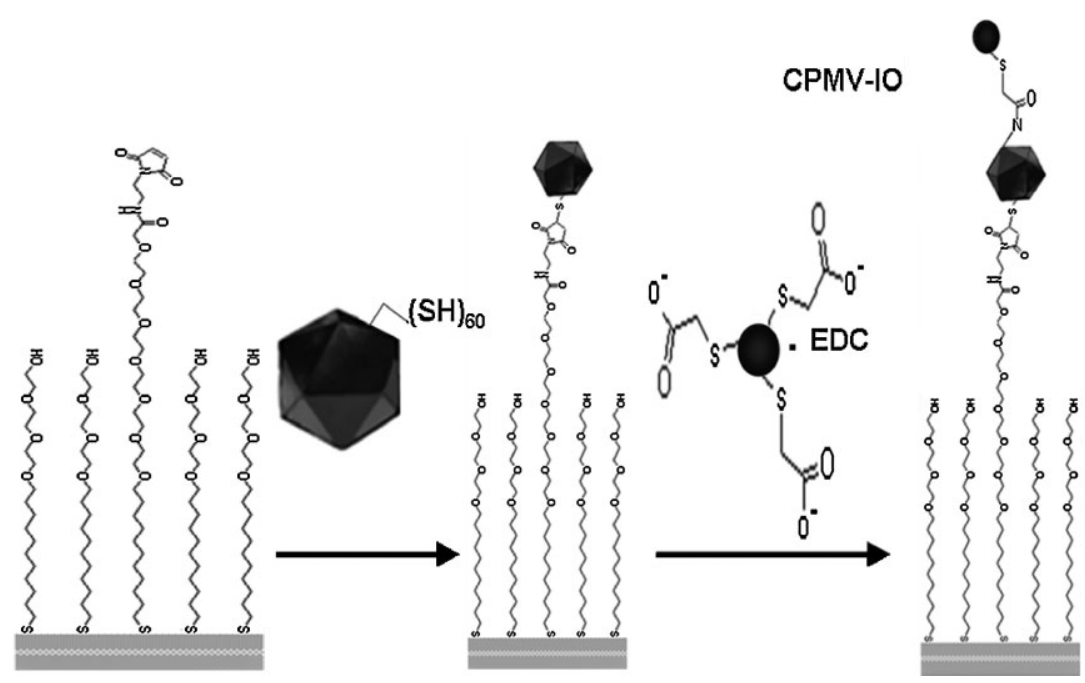

Figure 1. Stepwise substrate assembly of CPMV-IO hybrid.

TEM was used to characterize the viral surface morphologies before (inset Fig. 2B) and after (Fig. 2B) integration of IO nanoparticles onto the viral capsid of monodisperse CPMV-T184C mutants. In Figure 2B, the formation of CPMV-IO hybrids can be observed. The structural integrity and spatial organization of IO nanoparticles on the surface of the virions demonstrate the decoration of IO nanoparticles on CPMV-T184C virions.

Infrared spectroscopy by FTIR supports covalent attachment of CPMV-IO hybrids. Additional amide II bond character due to $\mathrm{C}-\mathrm{N}$ stretches are dominant in the hybrid $\left(1652 \mathrm{~cm}^{-1}\right)$ and amide I $\left(1648 \mathrm{~cm}^{-1}\right)$ by $\mathrm{C}=\mathrm{O}$ stretches (Fig. 3A). These vibrational modes are due to the resonating peptide backbone of the CPMV-T184C capsid, ${ }^{[21]}$ with amide II $(\mathrm{C}-\mathrm{N})$ contributed by condensation of carboxylated $\mathrm{IO}$ and CPMV-T184C lysines. CPMV-T184C reveals single amide I stretch at $1647 \mathrm{~cm}^{-1}$, a characteristic N-H stretch region (3500-3000 $\left.\mathrm{cm}^{-1}\right)$, and a small RNA peak at $1077 \mathrm{~cm}^{-1}$ (Fig. 3B). Carboxylated IO shows carbonyl $\mathrm{C}=\mathrm{O}$ stretch at

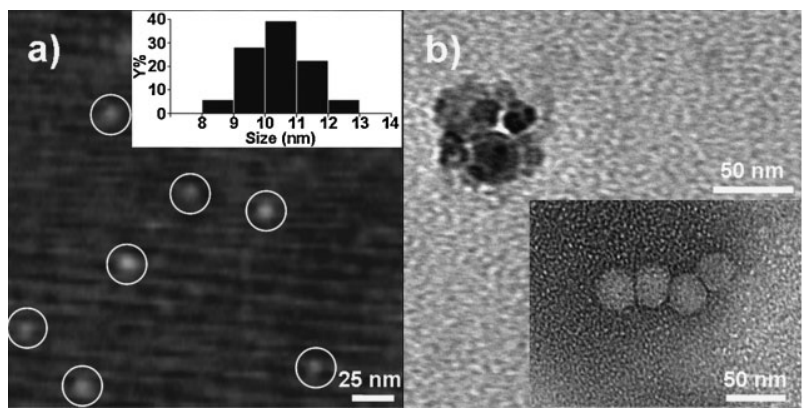

Figure 2. Characterization of $1 \mathrm{O}$ nanoparticles, CPMV-T184C, and CPMV-IO hybrids. a) AFM topography of as synthesized single IO nanoparticles (white circles). Inset shows the histogram of the measured size distribution for the single $1 \mathrm{O}$ nanoparticles. b) TEM of CPMV-IO hybrids recovered after thermal lift-off. Inset shows TEM of individual CPMV-T184C virions.
$1647 \mathrm{~cm}^{-1}$ (Fig. 3C). Spectra were obtained using $2 \mathrm{~cm}^{-1}$ resolution within blank $\mathrm{AgCl}$ windows as the background.

Atomic force microscopy in tapping mode (AFM) and MFM were used to study the topography and magnetic force gradient $\left(F^{\prime}=\partial F_{z} / \partial z\right)$ along the $z$-axis, respectively. The textured regions observed on each hybrid (Fig. 4A, white selections) are indicative of IO nanoclusters decorating the surface of single virions. Because the two-dimensional array of CPMV-IO hybrids was deposited onto the substrate in a disordered manner, the topography images show very large particles ( $\sim 200 \mathrm{~nm})$ in the background, consistent with gold grains on the silicon substrate. As discussed above, individual IO nanoparticles were observed and measured to have a nominal size of $\sim 11 \mathrm{~nm}$ (Fig. 2A). Therefore, in this work it is presumed that each observed IO nanocluster is in fact composed of several single IO nanoparticles. Due to the convolution of the tip with the closely packed individual nanoparticles, these cannot be clearly differentiated from each other but are rather imaged as a nanocluster.

Because in this work the substrate integration of a novel nanoparticle hybrid assembly was developed, it was essential to analyze the morphology to clearly show that two different nanomaterials are integrated in each hybrid. Figure $4 \mathrm{~B}$ provides in greater detail the morphology of a single CPMV-IO hybrid. The cross section (inset Fig. 4B) shows the particle features of CPMV to be $\sim 60 \mathrm{~nm}$, while the IO nanocluster is observed at $\sim 30 \mathrm{~nm}$ decorating the surface of the virion. To verify this decoration as a uniquely different nanomaterial system (e.g., IO), phase detection (Fig. 4C) was used. The difference in probe/sample interaction between the organic (CPMV) and inorganic (IO nanocluster) phase of the hybrid revealed that the visco-elastic properties of the IO nanoclusters are essentially different from those of CPMV. The scanning probe experiences a repellent force by the inorganic phase compared to an attractive force by the organic phase (inset Fig. 4C). Although the IO nanocluster is perceived by the probe as a repulsive force in the AFM phase detection, the magnetic force gradient image

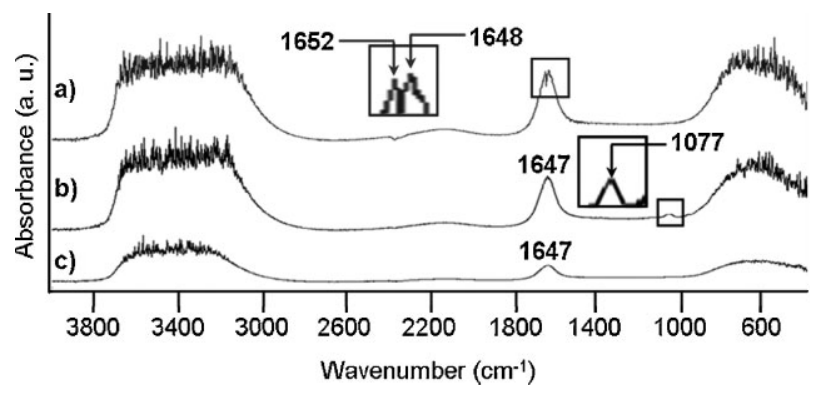

Figure 3. FTIR spectroscopy of CPMV-IO hybrids. a) Spectra of CPMV-IO hybrids in PBS solution ( $\mathrm{pH}=7.5)$. b) Spectra of CPMV-T184C. c) Carboxylated $\mathrm{Fe}_{2} \mathrm{O}_{3}$ (check) in PBS solution $(\mathrm{pH}=7.5)$. 


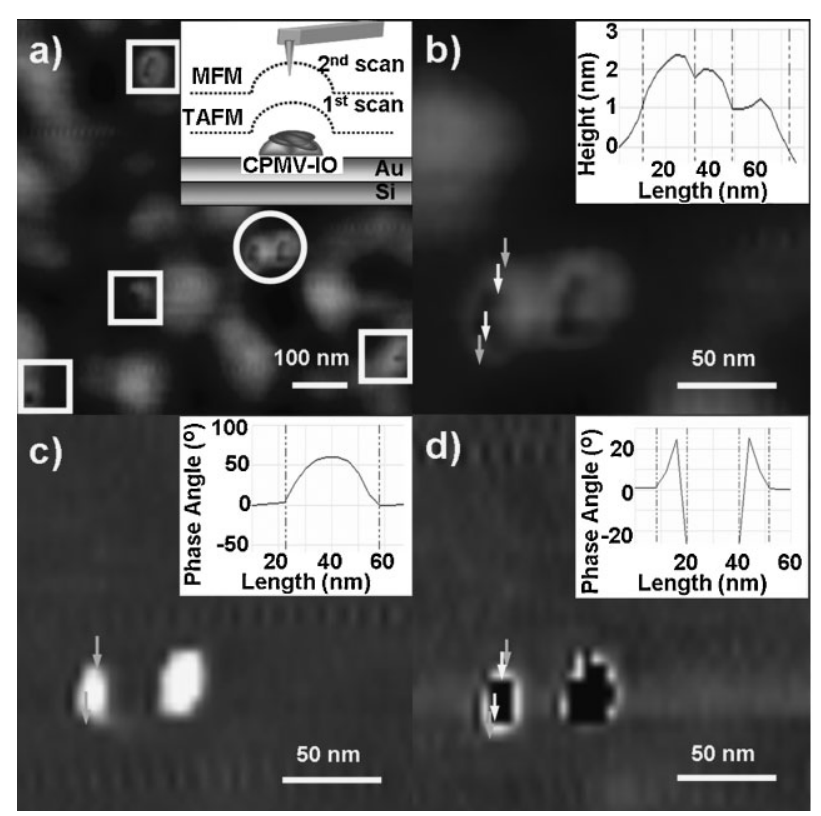

Figure 4. AFM and MFM imaging of single CPMV-IO hybrids. a) AFM topography showing single hybrids (whites squares). AFM/MFM schematic of dynamic lift-mode operation (inset). b) AFM topography, c) AFM phase detection, d) MFM phase detection of two adjacent CPMV-IO hybrids (white circle Fig. 3a) and their corresponding cross-sections (insets).

(Fig. 4D) shows a very interesting result in which a 'boundaryeffect' can be observed. MFM measurements show that as the magnetic probe scans the surface of a single hybrid, a repellent force is first exerted on the magnetic probe at the perimeter of the IO cluster. Then, as the probe moves away from the perimeter and into the inside region of each IO nanocluster a strong magnetic field gradient is measured by the probe and an attractive force is experienced by the cantilever (inset Fig. 4D). A similar "cluster edge effect" was observed by Pedreschi et al., ${ }^{[22]}$ in the characterization and simulation of colloidal iron nanoparticles. This boundary effect can be hypothesized to be likely due to a symmetry-breaking effect at the perimeter of the IO nanoclusters.

Furthermore, when MFM measurements were performed on hybrids with single IO nanoparticles on their surface, the strong magnetic force gradient measured on hybrids containing nanoclusters could not be observed. Figure 5A shows a sample area exhibiting both types of hybrids; hybrids with single IO nanoparticles (circles) and hybrids containing IO nanoclusters (squares) on their surface. The cross-section of a hybrid (enclosed by a dark circle in Fig. $5 \mathrm{~A}-\mathrm{C}$ ) with a single IO nanoparticle shows the size of the nanoparticle to be $\sim 12 \mathrm{~nm}$ (Inset Fig. 5A). Meanwhile, no major difference can be observed on the probe interaction with hybrids containing single IO nanoparticles during AFM/MFM phase detection measurements (Fig. 5B and $\mathrm{C}$, respectively). Cross-sections of a single hybrid during AFM/MFM phase detection measurements (Inset Fig. 5B and $\mathrm{C}$, respectively) shows that in both cases the probe experiences a slight repulsive force when scanning over the IO nanoparticle. The measured force is comparable in both cases and fundamentally of the same repulsive nature due to the inorganic phase of the hybrid. In addition, Figure $5 \mathrm{C}$ shows very clearly that only when the magnetic probe interacts with the hybrids containing IO nanoclusters a strong magnetic field gradient is measured during MFM. This data elucidates the notion that the local magnetic field strength of this hybrid system can be enhanced by covalently attaching (aggregating) IO nanoparticles onto the surface of CPMV-T184C mutants.

During AFM/MFM characterization it was observed that the ratio of hybrids with single IO nanoparticles to hybrids containing IO nanoclusters was 1 to 5. Figure 6 shows the histograms of the size distribution of IO nanoparticles (Fig. 6A) and IO nanoclusters (Fig. 6B) for both types of hybrids observed in this work. The measured size of single IO nanoparticles measured by AFM on the CPMV-IO hybrids is consistent with the size measured of the as synthesized IO nanoparticles before covalent attachment. The results presented here demonstrate the feasibility of covalently attaching IO nanoclusters on an organic scaffold (via substrate based integration) as a mean to enhancing the local magnetic field strength of IO nanoparticles.

To conclude, the enhanced local magnetic field strength was qualitatively analyzed by MFM, demonstrating a characteristic advantage for attaching derivatized magnetic IO nanoparticles in an organic medium. During MFM characterization a 'boundary-effect' was observed at the CPMV/IO interface. A strong magnetic field gradient was measured by the probe and the cantilever experienced a strong attractive force during MFM measurements. This strong interaction at a lift-off distance of $65 \mathrm{~nm}$ was indicative of a strong local magnetic field most likely due to a cumulative dipole effect of several IO nanoparticles clustered together. Such assembly processes are desirable in tailoring the physical-magnetic properties of a mutant hybrid which could provide multifunctional nanoparticles for enhanced MRI imaging.

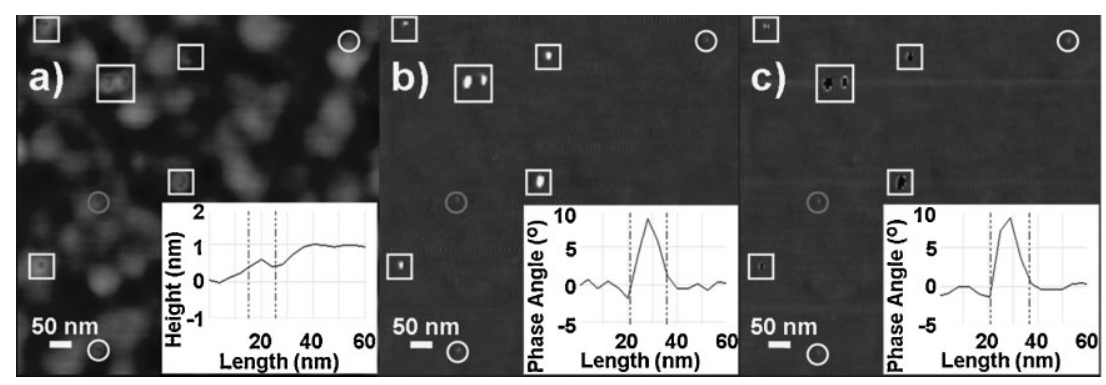

Figure 5. AFM and MFM imaging of CPMV-IO hybrids. a) AFM topography, b) AFM phase detection and c) MFM phase detection showing hybrids with single $I O$ nanoparticles (circles) and IO nanoclusters (squares) on their surface. Inset in figures a)-c) show the cross-section of single IO nanoparticle on the surface of a single virion (dark circle) as measured by AFM topography, AFM phase detection and MFM phase detection, respectively. 

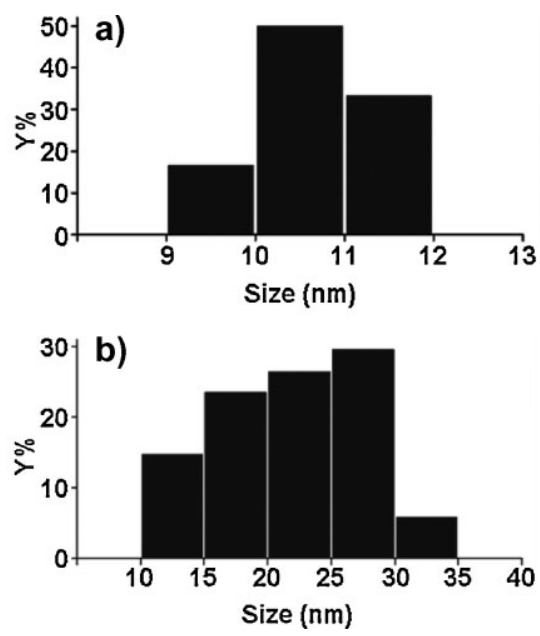

Figure 6. Histogram of the measured size distributions of a) single 10 nanoparticles and b) 10 nanoclusters covalently attached to the surface of CPMV-T184C as measured by AFM characterization.

\section{Experimental}

The chemicals, iron pentacarbonyl(80-90\%, Aldrich), octyl ether (99\%, Aldrich), oleic acid (90\%, Aldrich), 3-mercaptopropionic acid ( $99+\%$, Acros) and ethanol (200\% proof) and all other chemicals used for this study were of analytical grade and used with no further purification. The synthesis of $11 \mathrm{~nm}$ size IO nanoparticles was carried out under nitrogen atmosphere using standard schlenk technique. The biocompatible $\gamma-\mathrm{Fe}_{2} \mathrm{O}_{3}-\mathrm{COOH}$ nanoparticles were synthesized by thermal decomposition of $\mathrm{Fe}(\mathrm{CO})_{5}$ and surface modified as described in detail by Woo et al. [23]. IO nanoparticles protected by oleic acid where synthesized in a $100 \mathrm{~mL}$ volume schlenk flask, where oleic acid $(1.92 \mathrm{~mL}, 6.08 \mu \mathrm{mol})$ and octyl ether $(20 \mathrm{~mL})$ were taken and heated up to $100{ }^{\circ} \mathrm{C}$ and iron pentacarbonyl $(0.40 \mu \mathrm{mol}, 3.04 \mu \mathrm{mol})$ was added and continued to reflux for $2 \mathrm{~h}$. The reaction mixture was aerated for $14 \mathrm{~h}$ at $80^{\circ} \mathrm{C}$ and refluxed for another $2 \mathrm{~h}$. In order to obtain the Fe rich surface, nitrogen gas was bubbled into the reaction mixture for $2 \mathrm{~h}$ and iron pentacarbonyl $(0.04 \mathrm{~mL}, 0.30 \mu \mathrm{mol})$ was added at $100^{\circ} \mathrm{C}$ and continued to reflux for $1 \mathrm{~h}$. The above reaction mixture was cooled down to ambient temperature. 3-Mercaptopropionic acid $(0.053 \mathrm{~mL}$, $0.61 \mu \mathrm{mol}$ ) was added to the above mixture and refluxed for $1 \mathrm{~h}$. After cooling down to ambient temperature, excess ethanol was added to isolate IONPs-MPA by magnetic decantation. The mixture of chloroform/methanol/water was added to eliminate the excess surfactant. Pure product of IO nanoparticles tangled with 3-mercaptopropionic acid was isolated using a magnet.

CPMV-T184C was generated by site directed mutagenesis as described by Portney et al. [24]. The chimeric CPMVs were purified from the infected leaves by standard methods described by Dalsgaard et al., with some modifications [25]. Frozen leaves were homogenized in a Waring blender using $0.1 \mathrm{M}$ potassium phosphate $\mathrm{pH} 7.0,0.5 \%$ $\beta$-mercaptoethanol. After low-speed centrifugation, chloroformbutanol 1:1 was added to the supernatant and stirred for $20 \mathrm{~min}$ at $4{ }^{\circ} \mathrm{C}$. The aqueous phase was separated by centrifugation and the virus was precipitated using $8 \%(\mathrm{w} / \mathrm{v})$ polyethylene glycol 8000 and $0.2 \mathrm{M}$ $\mathrm{NaCl}$, stirring for $30 \mathrm{~min}$ at $4{ }^{\circ} \mathrm{C}$. After centrifugation the virus pellet was resuspended in $0.1 \mathrm{M}$ potassium phosphate $\mathrm{pH} 7.0$ and $0.2 \mathrm{M}$ DTT, and was layered on top of a $30 \%$ sucrose solution for ultracentrifugation $\left(42,000 \mathrm{rpm}, 3 \mathrm{~h}\right.$ at $\left.4{ }^{\circ} \mathrm{C}\right)$. The pellet was re-suspended in $3-5 \mathrm{~mL}$ of $0.1 \mathrm{~m}$ phosphate buffer at $\mathrm{pH} 7.0$ containing $0.1 \mathrm{~m}$ DTT. The CPMV particles were further purified on $10-40 \%$ sucrose gradients $\left(28,000 \mathrm{rpm}\right.$ for $3 \mathrm{~h}$ at $\left.4^{\circ} \mathrm{C}\right)$. Finally the virus was concentrated and any residual DTT was removed by ultrapelleting the virus through a $30 \%$ sucrose cushion $\left(42,000 \mathrm{rpm}\right.$ for $3 \mathrm{~h}$ at $\left.4{ }^{\circ} \mathrm{C}\right)$. CPMV-T184C contains 60 copies each of $\mathrm{L}$ and $\mathrm{S}$ proteins, with external lysines found at positions 38 and 82 on the small subunit and 34, 99, and 199 on the large subunit, for a total of 300 external lysines per capsid. Cysteines are inserted at position 184 of small subunit, achieving 60 total terminal cysteines per virus.

Deposition of maleimide disulfide/hydroxyl-capped disulfide alkanethiol (MED/EG3-EG3) species was performed by addition of ethanol washed and $\mathrm{N}_{2}$ gassed $\mathrm{Au}$ substrate (Platypus Technologies) into $1 \mathrm{~mm}$ MED/EG3-EG3 solution $(\sim 2 \mathrm{~mL})$ containing $1.1 \mathrm{~mm}$ total disulfide for up to $18 \mathrm{~h}$, followed by another ethanol wash and $\mathrm{N}_{2}$ drying. MED/EG3-EG3 coated Au substrates were submerged in a CPMV-T184C stock $\left(5 \mathrm{mg} \mathrm{mL}^{-1}\right)$ containing $2 \mathrm{~mm}$ tris(carboxyethyl)phosphine hydrochloride (TCEP) reducing agent for $1.5 \mathrm{~h}$ at RT. Following incubation, removed substrates were successively rinsed in PBS, Tween-20 (1 wt \%), and DI water, followed by $\mathrm{N}_{2}$ gas drying and storage in vacuum. Short reaction times in the presence of TCEP are used to prevent aggregation and saturation of CPMV on substrate, and avoid nonspecific adsorption effects to achieve a homogeneous sample.

Carbodiimide chemistry was used to condense solvent exposed primary amine lysine residues on CPMV-T184C with carboxylated IO [26]. To a solution of IO in PBS buffer $(\mathrm{pH}=7.5), 50 \mathrm{~mm}$ EDC (1-ethyl3-(3-dimethylaminopropyl)carbodiimide hydrochloride) was added to form a highly reactive $\mathrm{O}$-acylisourea intermediate for $15 \mathrm{~min}$, followed by $15 \mathrm{~min}$ ester formation with $5 \mathrm{~mm}$ sulfo-NHS (N-hydroxysulfosuccinimide) to extend the half life of the carboxylate to hours [27]. Following carboxylate activation, CPMV-T184C coated $\mathrm{Au}$ substrate was submersed for $4 \mathrm{~h}$ with gentle stirring. Following reaction, substrate was washed with PBS, Tween, and two DI washes before $\mathrm{N}_{2}$ drying and storage in vacuum.

CPMV-IO nanoparticles hybrids on Au substrate were desorbed by thermal lift off at $50{ }^{\circ} \mathrm{C}$ for $30 \mathrm{~min}$ in $500 \mathrm{uL}$ DI water. Lift off sample $\left(0.044 \mathrm{mg} \mathrm{mL}^{-1}\right)$ was added to plasma oxidized grid using Harrick PDC-326 plasma cleaner. TEM analysis was performed on 400 mesh plain carbon support film on $\mathrm{Cu}$ TEM grid (EMS, cat\#CF400-Cu) at $100 \mathrm{kV}$ accelerating voltage (FEI-Philips CM300). FTIR spectra were obtained by wetting an $\mathrm{AgCl}$ window with each sample into instrument (Bruker Equinox 55 FTIR spectrometer). OPUS spectroscopy software was used with $1 \mathrm{~cm}^{-1}$ resolution, where a PBS background averaged 20 scans. A/MFM experiments were performed with a Multimode V SPM system (Veeco Instruments Inc.). During experimental measurements a magnetic probe (MESP $\mathrm{Co} / \mathrm{Cr}$ silicon coated tip, $\mathrm{ROC} \sim 25 \mathrm{~nm}$ ) with a resonant frequency $60-100 \mathrm{kHz}$ was used. The nominal spring constant of the cantilever is $2.8 \mathrm{~N} \mathrm{~m}^{-1}$ with coercivity $\sim 400$ Oe. MFM measurements were performed in a dynamic lift-mode operation (inset Fig. 3A) at a lift-off distance of $65 \mathrm{~nm}$ over the sample, which was optimized in order to reduce the topographical interference and prevent false imaging (i.e., non-magnetic) of the CPMV-IO hybrids. All measurements were taken under open environment and ambient conditions.

Received: November 18, 2007

Revised: March 31, 2008

Published online: August 27, 2008

[1] A. S. Blum, C. M. Soto, C. D. Wilson, J. D. Cole, M. Kim, B. Gnade, A. Chatterji, W. F. Ochoa, T. Lin, J. E. Johnson, B. R. Ratna, Nano Lett. 2004, 4, 867.

[2] K. S. Raja, Q. Wang, M. J. Gonzalez, M. Manchester, J. E. Johnson, M. G. Finn, Biomacromolecules 2003, 4, 472.

[3] Q. Wang, T. Lin, J. E. Johnson, M. G. Finn, Chem. Biol. 2002, 9, 813. 
[4] J. M. Perez, T. O'Loughin, F. J. Simeone, R. Weissleder, L. Josephson, J. Am. Chem. Soc. 2002, 124, 2856.

[5] A. Dyal, K. Loos, M. Noto, S. W. Chang, C. Spagnoli, K. V.P. M. Shafi, A. Ulman, M. Cowman, R. A. Gross, J. Am. Chem. Soc. 2003, 125, 1684.

[6] C. Alexiou, A. Schmidt, R. Klein, P. Hulin, C. Bergemann, W. Arnold, J. Magn. Magn. Mater. 2002, 252, 363.

[7] P. Moroz, H. Pardoe, S. K. Jones, T. G. St Pierre, S. Song, B. N. Gray, Phys. Med. Biol. 2002, 47, 1591.

[8] P. R. Clark, W. Chua-anusorn, T. G. St Pierre, Magn. Reson. Med. 2003, 49, 572 .

[9] L. Babes, B. Denizot, G. Tanguy, J. J. Le Jeune, P. Jallet, J. Colloid Interface Sci. 1999, 212, 474.

[10] R. Hergt, W. Andra, C. G. d'Ambly, I. Hilger, W. A. Kaiser, U. Richter, H.-G. Schmidt, IEEE Trans. Magn. 1998, 34, 3745.

[11] H. Lee, E. Lee, D. K. Kim, N. K. Jang, Y. Y. Jeong, S. Jon, J. Am. Chem. Soc. 2006, 128, 7383 .

[12] A. K. Gupta, S. Wells, IEEE Trans. Nanobiosci. 2004, 3, 66.

[13] T. J. Harris, G. von Maltzahn, A. M. Derfus, E. Ruoslahti, S. N. Bhatia, Angew. Chem. Int. Ed. 2006, 45, 3161.

[14] T. Lin, Z. Chen, R. Usha, C. V. Stauffacher, J.-B. Dai, T. Schmidt, J. E. Johnson, Virology 1999, 265, 20.

[15] Q. Wang, E. Kaltgrad, T. Lin, J. E. Johnson, M. G. Finn, Chem. Biol. 2002, 9, 805 .
[16] N. G. Portney, R. J. Tseng, G. Destito, E. Strable, Y. Yang, M. Manchester, M. G. Finn, M. Ozkan, Appl. Phys. Lett. 2007, 90, 214104.

[17] H. Maeda, T. Sawa, T. Konno, J. Controlled Release 2001, 74, 47.

[18] D. P. O'Neal, L. R. Hirsch, N. J. Halas, J. D. Payne, J. L. West, Cancer Lett. 2004, 209, 171.

[19] T. R. Green, J. Fisher, M. Stone, B. M. Wroblewski, E. Ingham, Biomaterials 1998, 19, 2297.

[20] S. M. Moghimi, A. C. Hunter, J. C. Murray, Pharmacol. Rev. 2001, 53, 283.

[21] H. H. Mantsch, D. Chapman, Infrared Spectroscopy of Biomolecules, Wiley, New York 1996, p. 239.

[22] F. Pedreschi, J. M. Sturm, J. D. O’Mahony, C. F. J. Flipse, J. Appl. Phys. 2003, 94, 3446.

[23] K. Woo, J. Hong, IEEE Trans. Magn. 2005, 41, 4137.

[24] N. G. Portney, A. A. Martinez-Morales, M. Ozkan, ACS Nano 2008, 2, 191.

[25] K. Dalsgaard, A. Uttenthal, T. D. Jones, F. Xu, A. Merryweather, W. D. O. Hamilton, J. P. M. Langeveld, R. S. Boshuizen, S. Kamstrup, G. P. Lomonossoff, C. Porta, C. Vela, J. I. Casal, R. H. Meloen, P. B. Rodgers, Nat. Biotechnol. 1997, 15, 248.

[26] H. G. Khorana, Chem. Rev. 1953, 53, 145.

[27] G. T. Hermanson, Bioconjugate Techniques, Academic, San Diego, CA 1996. 